\title{
COVID-19 and Cardiovascular Diseases: Challenges and Solutions
}

\author{
Prachi Sharma $^{\mathrm{a}}$, Akshyaya Pradhan ${ }^{\mathrm{a}, \mathrm{b}}$, Shweta Vohra ${ }^{\mathrm{a}}$, Rishi Sethi ${ }^{\mathrm{a}}$
}

\begin{abstract}
Rampantly spreading around the globe and taking thousands of lives each day, coronavirus disease 2019 (COVID-19) may present with varied cardiovascular manifestations. Those with evidence of myocardial injury have a worse prognosis too. Patients with preexisting cardiovascular diseases or risk factors are at greater risk of adverse outcomes and mortality by this infection. Being highly contagious, measures to prevent cross-infection are of paramount importance. In this article, the authors summarize the various cardiovascular manifestations of COVID-19 and precautions needed while handling them and the long-term consequences of COVID-19 infection.
\end{abstract}

Keywords: Coronavirus; Pandemic; Myocarditis; Inflammation

\section{Introduction}

With incessant advances in science and technology, cardiology has seen the most rapid advances in the last three decades. In the midst of this, the world is now facing the worst pandemic of the century. With downfall and again a surge of cases worldwide, according to the World Health Organization's daily report, this third zoonotic coronavirus disease pandemic, coronavirus disease 2019 (COVID-19) by severe acute respiratory syndrome coronavirus 2 (SARS-CoV-2) has affected more than 110 million people and claimed more than 2,040,000 lives across the world as on February 23, 2020. Although the most common presenting symptoms are fever, cough and malaise, several reports have seen varied manifestations including myocardial ischemia and heart failure [1]. Also, the prognosis of COVID-19 patients is worse when there is accompanying myocardial injury [2].

Manuscript submitted February 23, 2021, accepted March 9, 2021

Published online May 14, 2021

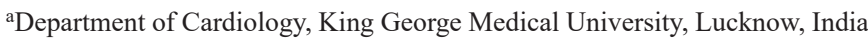
${ }^{b}$ Corresponding Author: Akshyaya Pradhan, Department of Cardiology, King George Medical University, Lucknow 226003, India.

Email: akshyaya33@gmail.com

doi: https://doi.org/10.14740/cr1244

\section{COVID-19 in Patients With Pre-Existing Cardio- vascular Diseases}

Patients with pre-existing cardiovascular diseases and comorbidities like diabetes and hypertension are at increased risk of worse outcomes following COVID-19 [3]. In a cohort of 191 patients from Wuhan, China, $48 \%$ (67\% of non-survivors) had some comorbidity, hypertension in $30 \%$ ( $48 \%$ of non-survivors), diabetes mellitus (DM) in 19\% (31\% of non-survivors), and cardiovascular diseases (CVD) in $8 \%$ (13\% of non-survivors) [4]. In another cohort of 138 hospitalized COVID-19 patients [5] from Wuhan, comorbidities were more prevalent in patients requiring intensive care unit (ICU) care (46\% overall and $72 \%$ in patients requiring ICU care), hypertension in $31 \%$ (58\% in patients requiring ICU care), CVD in $15 \%$ (25\% in patients requiring ICU care), and DM in $10 \%$ (22\% in patients requiring ICU care).

\section{Acute Myocardial Injury in COVID-19}

Apart from the respiratory system involvement, COVID-19 presents with cardiovascular manifestations too. In a pooled analysis of 11,685 patients [6], the prevalence of acute myocardial injury was $20 \%$ (ranging from $5 \%$ to $38 \%$ according to the criteria used. Several mechanisms for cardiovascular involvement have been proposed: 1) Direct viral invasion of the myocardium [7]; 2) Excessive immune mediated response to the virus causing myocarditis [8]; 3) Hypoxia of cardiac myocytes as a result of respiratory failure; 4) Hypercoagulable state induced by the SARS-CoV-2 may result in coronary microvascular thrombosis [9]; 5) Type I myocardial infarction (MI) as a result of stress related plaque rupture; 6) Type II MI due to demand-supply mismatch in stressful state; 7) Stress cardiomyopathy; 8) Down-regulation of angiotensin-converting enzyme 2 (ACE-2) receptors resulting in possible reduced protective signaling [10]. Figure 1 enumerates the various mechanism of COVID-19 induced myocardial damage.

While the immune system tries to fight the invading virus, excessive immune mediated inflammatory response may cause myocarditis. On multivariate adjusted analysis in a study of 182 COVID-19 patients by Li et al [11], age, whole blood cell count, neutrophil percentage, lymphocyte percentage, cluster of differentiation $(\mathrm{CD}) 3^{+} \mathrm{T}$ cell counts, $\mathrm{CD} 4^{+} \mathrm{T}$ cell counts, $\mathrm{CD}^{+} \mathrm{T}$ cell counts, $\mathrm{CD} 16^{+} / \mathrm{CD}^{2} 6^{+}$(natural killer) NK cell 


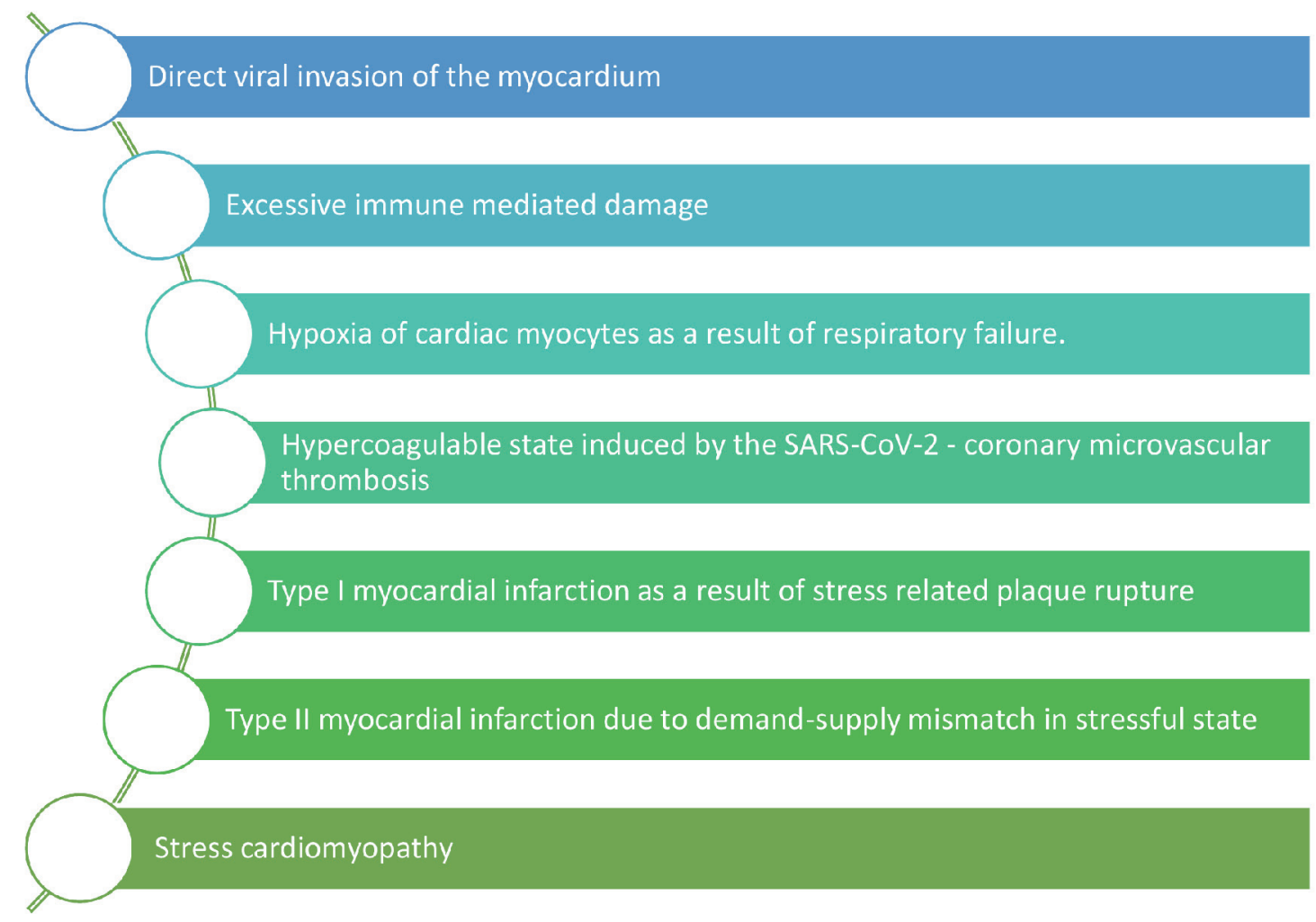

Figure 1. Various mechanisms of myocardial damage by severe acute respiratory syndrome coronavirus 2 (SARS-CoV-2) infection leading to cardiac manifestations.

counts, high-sensitivity (hs)-C-reactive protein, and procalcitonin were independently associated with myocardial injury in patients with COVID-19.

The ACE-2 receptors being found on the epithelial cells of lungs, heart, kidneys, intestines and vascular endothelium are used by SARS-CoV-2 for entry into target cells. This may cause direct cardiac myocyte injury [10]. Furthermore, SARS-CoV-2 binding to ACE-2 leads to its downregulation and increases angiotensin II. This leads to lesser amounts of angiotensin (17). This causes angiotensin type 1 receptor (AT1R)-mediated systemic vasoconstriction and increased pulmonary vascular permeability.

Cardiac biomarkers are most helpful for diagnosis as well as for prognostic implications [2]. In a study of 671 COVID-19 patients by Shi et al [12], the prevalence of myocardial injury defined as hs-troponin I above the 99th percentile was $15.8 \%$. Both CK-MB $>2.2 \mathrm{ng} / \mathrm{mL}$ (hazards ratio, 6.62, $\mathrm{P}<0.001$ ) and cardiac troponin $\mathrm{I}(\mathrm{cTnI})>0.026 \mathrm{ng} / \mathrm{mL}$ (hazards ratio, 4.56, $\mathrm{P}=0.02$ ) were found to be independently associated with increased in-hospital mortality. In a multicentre study analysing 68 fatal cases of COVID-19 [13], myocardial injury with or without respiratory failure was noted to be the cause of death in $40 \%$ of cases. In a report of 138 COVID-19 patients hospitalized at Zhongnan University Hospital of Wuhan, China, cardiac injury (elevated hs-cTnI or new electrocardiographic or echocardiographic abnormalities) was present in $7.2 \%$ of patients overall and $22 \%$ of patients requiring ICU care [5].

While the World Health Organization document (Clinical management of severe acute respiratory infection when COVID-19 disease is suspected (version 1.2, March 13, 2020)) [14] recommends laboratory testing for acute myocardial injury at admission and as clinically indicated, the American College of Cardiology (ACC) advices to measure troponin only if the diagnosis of acute MI is being considered on clinical grounds [15].

\section{Cardiovascular Manifestations of COVID-19}

Cardiovascular manifestations of COVID-19 can range from asymptomatic elevation of cardiac injury biomarkers to fulminant myocarditis and circulatory shock (Fig. 2). Table 1 [4, 5, $12,16]$ quotes the incidence of various cardiovascular presentations of COVID-19 in published literature. It may be difficult at times to clearly define whether the elevated cardiac biomarkers are due to myocardial ischemia, myocarditis, impaired renal function, pulmonary embolism, or the systemic inflammatory state. So, troponin elevations need to be evaluated in conjunction with other inflammatory markers like serum ferritin and interleukin-6 (IL-6) to define the underlying etiology. But it is certain that these patients require the most intensive care.

\section{Acute coronary syndrome}

The concern of disease mimickers has grabbed even ST elevation MI (STEMI) and the reasons are genuine. Both STEMI 


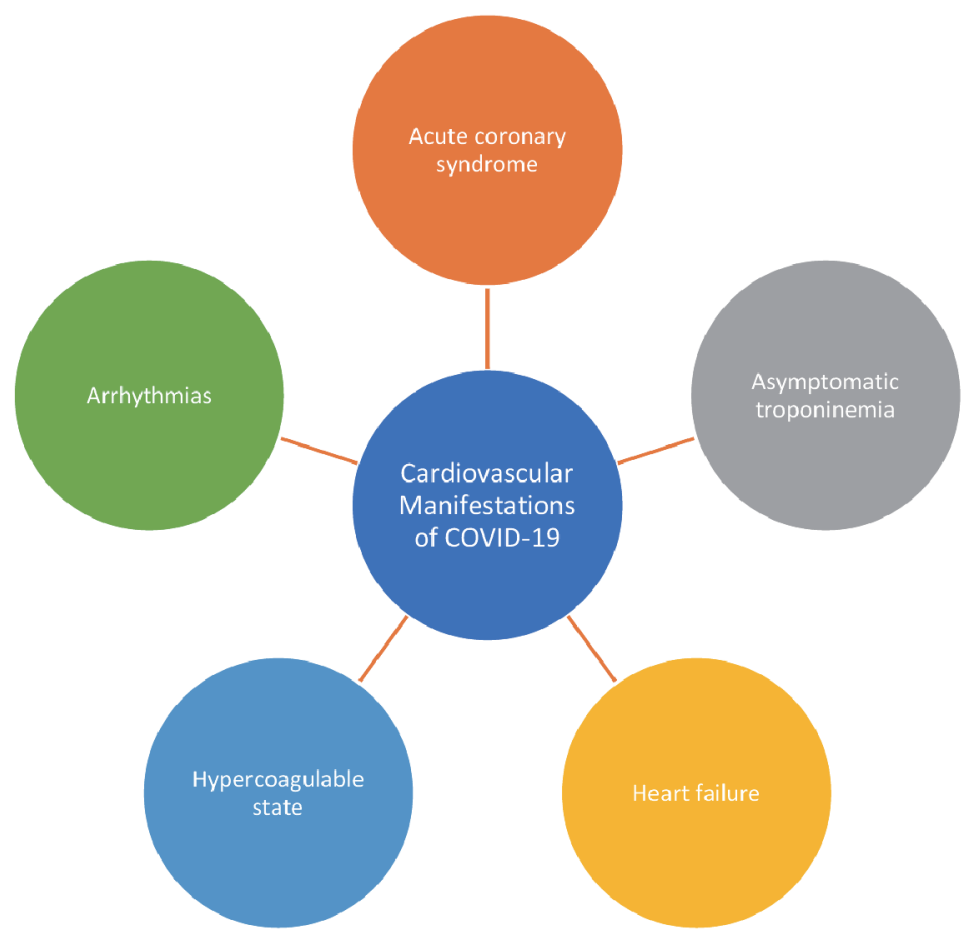

Figure 2. The varied cardiovascular manifestations of coronavirus disease 2019 (COVID-19) infection.

and non-STEMI are reported in patients hospitalized with COVID-19. There are case reports where patients presented with STEMI but no other apparent clinical manifestation of viral illness. A single-centre retrospective analysis from Northern Italy [17] of 28 patients with COVID-19 and STEMI found no obstructive coronary artery disease in around $40 \%$ patients. Amongst them, in 24 patients (85.7\%) STEMI represented the first clinical manifestation of COVID-19 and did not have a COVID-19 test result at the time of coronary angiography. This may lead to delay in detection of COVID-19 and spread of infection. Also, several STEMI appearing presentations may in fact be COVID-19 associated myocarditis.

\section{Arrhythmias}

In studies by Wang and colleagues [5] and Goyal et al [18] including 138 and 393 consecutive hospitalized COVID-19 patients respectively, the incidence of arrhythmia was found to be $17 \%$ and $7.4 \%$, respectively. Although they did not describe the types of arrhythmias, both tachyarrhythmias and bradyarrhythmias can occur. In another study by Guo et al [19] of 187 COVID-19 patients, the incidence of ventricular arrhythmias was $5.9 \%$, and was significantly more common in patients with myocardial injury than in those without (17.3\% vs. $1.4 \%$, $\mathrm{P}<0.001)$.

\section{Heart failure}

In a study of 191 COVID-19 patients by Zhou et al [4], heart failure was observed in $23.0 \%$ of patients. Elderly patients with pre-existing hypertension or diastolic dysfunction are at greater risk of new onset heart failure. The cytokine surge may lead to worsening of pre-existing heart failure, development of myocarditis and left ventricular systolic dysfunction [20]. There may be pulmonary hypertension due to lung involvement by COVID-19 and subsequent right heart failure in substantial number of patients. This is further associated with increased in hospital mortality [21].

Table 1. The Incidence of Various Cardiovascular Presentations of COVID-19 in Published Literature

\begin{tabular}{|c|c|c|}
\hline Clinical manifestations & Incidence & Reference \\
\hline Increased cardiac troponin & $15.8 \%$ & {$[12]$} \\
\hline Acute cardiac injury & $17 \%$ overall ( $59 \%$ in non-survivors vs. $1 \%$ in survivors) & {$[4]$} \\
\hline Arrhythmias & $17 \%$ & {$[5]$} \\
\hline Hypercoagulable state & $71 \%$ in those who died and $0.6 \%$ in those who recovered & {$[16]$} \\
\hline
\end{tabular}




\section{Hypercoagulable state}

COVID-19 patients are found to have deranged coagulation profile. Hypercoagulable states resulting in deep venous thrombosis, pulmonary embolism, coronary intravascular thrombosis causing acute MI and cerebral thrombosis leading to stroke have been reported. Patients are found to have elevated D-dimer and mild thrombocytopenia most commonly, which is prognostic and associated with increased need for mechanical ventilation [22]. Retrospective study of $183 \mathrm{COV}$ ID-19 patients by Tang et al [16] found that $71 \%$ of COVID-19 patients who died fulfilled the International Society on Thrombosis and Hemostasis (ISTH) criteria for disseminated intravascular coagulation (DIC), compared with only $0.6 \%$ among survivors. They found significantly higher D-dimer and prothrombin time in non-survivors as compared to survivors. A variety of potential risk factors exist including immobilisation, heart failure, chronic kidney disease, chronic obstructive lung disease, malignancy, mechanical ventilation and central venous catheter use. Risk stratification tools may be used to predict venous thromboembolism risk in acutely ill hospitalized patients. Padua risk model used by a study in China showed that $40 \%$ of hospitalized patients with COVID-19 were at high risk of venous thromboembolism, $11 \%$ of whom also had high bleeding risk [23].

\section{The Impact on the Care of Cardiovascular Pa- tients}

The number of patients presenting to cardiology emergency room (ER) with STEMI has also dramatically fallen, leading to almost $40 \%$ reduction in catheterization laboratory activation [24]. People avoid seeking medical help despite chest pain for the fear of exposure to SARS-CoV-2. There is limited transportation available and limited access to medical facilities, which further hampers timely medical contact. Hospital resources are allocated to COVID-19 and several hospitals are unable to cater to other patients. The increase in late presenters of MI is leading to an increase in complications like ventricular septal rupture [25]. In the majority of the centers, all elective procedures are postponed. Worse is that there is no followup of patients and effects on patient progress or worsening is maximally unknown. It is a tough responsibility to make the public aware that they should seek appropriate cardiac care in the prevailing scenario too. This confidence in the general population can be built by taking appropriate precautions to avoid inadvertent cross exposure and still provide adequate treatment.

\section{Management of Cardiovascular Diseases}

It is essential to keep a broad view and still not ignore MI while dealing with elevated troponin levels in a COVID-19 patient. Although myocarditis and stress cardiomyopathy due to COVID-19 may mimic STEMI, it is important to recognize true STEMI and offer emergent management. While, the American
College of Cardiology (ACC) and Society for Cardiovascular Angiography and Intervention (SCAI) advocate primary percutaneous intervention (PCI) for definite STEMI [26], decisions need to be individualized according to the availability of resources. ACC defends primary PCI on the grounds of need for emergency rescue PCI in significant majority of patients following unsuccessful fibrinolysis. Also, the STEMI mimics when wrongly fibrinolysed lead to an increased bleeding risk. In case of diagnostic dilemma and less typical chest pain, echocardiography may be done by the same team without exposing additional staff. It may be reasonable to avoid catheterization laboratory activation if the patient is a late presenter. Opinions are diverse but in view of the community spread of COVID-19 and uncertain sensitivity of diagnostic tests, several societies are recommending the use of fibrinolytic therapy for all STEMI unless hemodynamic instability. This reduces the exposure of health care personnel and vice versa. A pharmacoinvasive approach may be recommended with initial fibrinolysis and testing for COVID-19 status unless rescue PCI is deemed necessary. COVID-19 positive or probable patients with non-STEMI may be managed medically and only taken for urgent coronary angiography and possible PCI in the presence of high-risk clinical features (Global Registry of Acute Coronary Events (GRACE) score > 140) or hemodynamic instability [27]. In patients in whom we anticipate the need for invasive ventilation or intra-aortic balloon pump (IABP), such procedures should be done bedside to avoid doing emergently in catheterisation laboratory. Disinfection of the instruments and catheterisation laboratory is essential after each case. Chlorine containing disinfectants are shown to be effective in clearing the viral load [28].

\section{Healthcare Personnel Protection}

While it is crucial to provide efficient patient care, it is of utmost importance to ensure safety of the health care personnel involved therein. This is challenging in the times of unforeseen pandemic when there is shortage of personal protective equipment. Catheterization laboratory personnel need to be provided with adequate personal protective equipment (PPE) to avoid cross contamination. The Center for Disease Control and Prevention recommends contact and droplet PPE for health care personnel in their routine care of patients with COVID-19. If an aerosol-generating procedure is to be performed like intubation and cardiopulmonary resuscitation, additional PPE with an N95 respirator is recommended. All elective procedures need to be postponed to save resources for the management of COVID-19 increasing number of patients. Also, the fear of contacting asymptomatic carriers is genuine. This calls for avoiding all outpatient follow-up visits and advocating telemedicine. While visiting ER, the patients and the attendants should wear mask and keep proper distancing.

\section{Drug Treatment and Interactions}

Awaiting definite evidences, several anti-viral and immune- 
modulatory agents are being tried to help patients recover. Although initial reports advocated the use of hydroxychloroquine (HCQ) and azithromycin for the treatment of patients with COVID-19, subsequent large scale studies have not found significant survival benefit. Remdesivir is a nucleotide analog that interrupts ribonucleic acid (RNA) replication. It has been found to improve survival with a 14-day mortality of $7.1 \%$ with remdesivir vs. $11.9 \%$ with placebo (hazard ratio 0.70 ; 95\% confidence interval (CI): 0.47 - 1.04) in the RECOVERY (Randomized Evaluation of COVid-19 thERapY) trial, which is one of the largest randomized clinical trials testing a range of potential treatments for COVID-19 [29]. The same trial recently showed that low dose oral/intravenous dexamethasone treatment prevented one death for around eight ventilated patients and around 25 patients requiring oxygen alone [30]. A total of 2,104 patients were randomized to receive dexamethasone and were compared with 4,321 patients randomized to usual care alone. Dexamethasone $6 \mathrm{mg}$ once per day reduced deaths by one-third in ventilated patients (rate ratio: 0.64 ; 95\% CI: 0.51 0.81 ), and by one-fifth in other patients receiving oxygen only (0.82 (0.72 - 0.94), $\mathrm{P}<0.001)$. There was no benefit among those patients who did not require respiratory support (1.19 $(0.92-1.55)$ [30].

Protease inhibitors lopinavir/ritonavir, antiviral oseltamivir used for influenza and ribavirin have been used, but controlled trials did not demonstrate statistically significant benefit [31]. Favipiravir, another drug approved for influenza treatment, is considered promising because it inhibits RNA polymerase. Tocilizumab, an IL-6 receptor antagonist, has been used with reported success in patients with severe COVID-19, and clinical trials are ongoing. A case report described how the left ventricular dysfunction and biomarkers normalized within 3 weeks of intravenous immunoglobulin and steroids in a COVID-19 patient presenting with chest pain and ST-segment elevation on his electrocardiogram but without coronary obstruction [32].

There has been considerable debate regarding the use of ACE inhibitor and angiotensin receptor blockers (ARB) in patients with COVID-19. As renin-angiotensin-aldosterone system inhibitors upregulate the expression of ACE-2 in tissues [10], it was initially thought to promote entry of COVID-19 into cells. But studies have found that increased expression of ACE-2 is rather protective. ACE-2 cleaves angiotensin II, a vasoconstrictor to angiotensin (1-7), which is a vasodilator. A retrospective analysis of 1,128 COVID-19 patients with a previous history of hypertension, concomitant in hospital use of ACE inhibitors or ARB was associated with lower risk of all-cause mortality compared to non-users [33]. Currently, it is recommended to continue $\mathrm{ACE}$ inhibitors and $\mathrm{ARB}$ in patients already taking them.

Being used by hundreds of millions of people worldwide since several decades as anti-malarial and for several rheumatological diseases, HCQ has been quite safe and even available over the counter in several countries. The possibility of QTc prolongation, torsades de pointes and sudden death becomes a concern with concomitant use of multiple QTc-prolonging drugs and/or the presence of ion disturbances (hypokalemia, hypomagnesemia and/or hypocalcemia) [34]. Chorin et al [35] showed in a population of 85 COVID-19 pa- tients that in 30\% QTe increased by more than $40 \mathrm{~ms}$ and $11 \%$ showed a severe prolongation (QTc $>500 \mathrm{~ms}$ ) while on treatment with HCQ and azithromycin. Despite that, none of them developed torsades de pointes. Saleh et al [36] also evaluated 201 COVID-19 patients who during hospitalization received chloroquine/HCQ as a monotherapy $(61 \%)$ or in association with azithromycin (59\%), 9\% of patients showed a QTc $>500$ ms on treatment (3.5\% had discontinuation of therapy). But no torsades de pointes or arrhythmic death were reported. It has also been seen in recent studies that HCQ started early in the course of illness and for longer duration reduced the severity of illness and prevented some hospitalizations; while, this was quite less effective if started late after disease progression [37].

Believed to have pulmonary vasodilator and anti-viral properties, there are certain ongoing trials on nitric oxide (NO) nasal irrigation, inhaled therapy and even oral administration vs. placebo on severity of illness in hospitalized patients. In a recent study on 34 patients with severe COVID-19, 65\% responded to inhaled NO therapy with reduction in pressure of arterial oxygen to fractional inspired oxygen concentration $\left(\mathrm{PaO}_{2} / \mathrm{FiO}_{2}\right)$ by more than $20 \%$ [38]. This may be a potential therapy in severe COVID-19 pneumonia patients with persistent hypoxemia. But due to lack of sufficient research data, it is still not being recommended. Another field of ongoing research is convalescent plasma therapy. In a recent study on patients with severe COVID-19 pneumonia [39], out of 334 patients enrolled, 228 received convalescent plasma while 105 were randomized to placebo. There was no significant benefit in terms of 30-day clinical status or overall mortality with convalescent plasma vs. placebo. While, another recent study on 180 subjects showed that if convalescent plasma is administered early in the course of illness, there is significant reduction in progression of severe COVID-19 respiratory illness by $48 \%$ [40]. This trial randomized patients with mild illness, within $72 \mathrm{~h}$ of onset of symptoms. This emphasizes that early administration of sufficient antibodies may prevent worsening of illness.

In view of increased venous thrombosis risk, anticoagulation remains the mainstay of prophylaxis and treatment. It needs to be emphasized that the patients at higher thromboembolic risk may also be at a higher bleeding risk. The duration and dosing of anti-coagulants need to be adjusted accordingly. Also, elastic compression stockings and intermittent pneumatic compression may be considered. Currently, it is recommended to anticoagulate all COVID-19 hospitalized patients preferably with low-molecular-weight heparin subcutaneous injection. At discharge, the National institute of Health (NIH) discourages routine thromboprophylaxis. However, it does advocate NOAC in patients at high risk of venous thromboembolism (VTE) based on D-Dimer levels and IMPROVE-VTE risk score.

\section{Long-Term Cardiovascular Sequelae}

Long-term consequences of COVID-19 may be caused by increased IL-6 signalling. Apart from cardiac fibrosis, IL-6 and 
other cytokines have been proposed to have role in atherogenesis [41]. Also, cardiac magnetic resonance imaging has demonstrated cardiac involvement in significant number of patients who had recovered from COVID-19 [42]. Whether this translates to long-term consequences or recovers spontaneously is still unknown.

\section{Conclusions}

Awaiting effective vaccines, personal preventive measures are the best strategy in COVID-19. Patients with pre-existing cardiovascular diseases or risk factor need to be extra cautious as they may be the worst hit. Lest the fear of catching COVID-19 infection prevail, one should know that untreated MI has even higher mortality. Lockdown resulting in unemployment, financial losses, loneliness, anxiety, depression and lack of physical activity is associated with anticipated increased risk of cardiovascular diseases. These consequences shall affect the lives of people even after the world comes out of this COVID-19 pandemic.

\section{Acknowledgments}

None to declare.

\section{Financial Disclosure}

None to declare.

\section{Conflict of Interest}

None to declare.

\section{Author Contributions}

AP conceived the project and did the literature search. PS prepared the main manuscript draft. RS critically reviewed the manuscript and suggested additional changes. AP added figures and PS prepared the final manuscript. AP did journal scan and PS submitted the final manuscript. SV provided the tables and was responsible for bibliography.

\section{Data Availability}

The authors declare that data supporting the findings of this study are available within the article.

\section{References}

1. Clerkin (KJ, Fried JA, Raikhelkar J, Sayer G, Griffin JM, Masoumi A, Jain SS, et al. COVID-19 and cardiovascular disease. Circulation. 2020;141(20):1648-1655.

2. Bonow RO, Fonarow GC, O'Gara PT, Yancy CW. Association of coronavirus disease 2019 (COVID-19) with myocardial injury and mortality. JAMA Cardiol. 2020;5(7):751-753.

3. $\mathrm{Wu} \mathrm{Z}$, McGoogan JM. Characteristics of and important lessons from the coronavirus disease 2019 (COVID-19) Outbreak in China: Summary of a report of 72314 cases from the Chinese Center for Disease Control and Prevention. JAMA. 2020;323(13):1239-1242.

4. Zhou F, Yu T, Du R, Fan G, Liu Y, Liu Z, Xiang J, et al. Clinical course and risk factors for mortality of adult inpatients with COVID-19 in Wuhan, China: a retrospective cohort study. Lancet. 2020;395(10229):1054-1062.

5. Wang D, Hu B, Hu C, Zhu F, Liu X, Zhang J, Wang B, et al. Clinical characteristics of 138 hospitalized patients with 2019 novel coronavirus-infected pneumonia in Wuhan, China. JAMA. 2020;323(11):1061-1069.

6. Bavishi C, Bonow RO, Trivedi V, Abbott JD, Messerli FH, Bhatt DL. Special Article - Acute myocardial injury in patients hospitalized with COVID-19 infection: A review. Prog Cardiovasc Dis. 2020;63(5):682-689.

7. Rasmussen A, Smulian JC, John A, Tony S, Wen MD, Denise J, Jamieso M. Endothelial cell infection and endotheliitis in COVID-19. Lancet. 2020.

8. Zhou Y, Fu B, Zheng X, Wang D. Zhao CH, Qi Y. Pathogenic $\mathrm{T}$ cells and inflammatory monocytes incite inflammatory storm in severe COVID-19 patients. Natl Sci Rev. 2020.

9. Han H, Yang L, Liu R, Liu F, Wu KL, Li J, Liu XH, et al. Prominent changes in blood coagulation of patients with SARS-CoV-2 infection. Clin Chem Lab Med. 2020;58(7):1116-1120.

10. Chen L, Hao G. The role of angiotensin-converting enzyme 2 in coronaviruses/influenza viruses and cardiovascular disease. Cardiovasc Res. 2020;116(12):1932-1936.

11. Li D, Chen Y, Jia Y, Tong L, Tong J, Wang W, Liu Y, et al. SARS-CoV-2-induced immune dysregulation and myocardial injury risk in China: insights from the ERSCOVID-19 study. Circ Res. 2020;127(3):397-399.

12. Shi S, Qin M, Cai Y, Liu T, Shen B, Yang F, Cao S, et al. Characteristics and clinical significance of myocardial injury in patients with severe coronavirus disease 2019. Eur Heart J. 2020;41(22):2070-2079.

13. Ruan Q, Yang K, Wang W, Jiang L, Song J. Clinical predictors of mortality due to COVID-19 based on an analysis of data of 150 patients from Wuhan, China. Intensive Care Med. 2020;46(5):846-848.

14. World Health Organization. Clinical management of severe acute respiratory infection when novel coronavirus (2019-nCoV) infection is suspected: interim guidance. In: Clinical management of severe acute respiratory infection when novel coronavirus $(2019-\mathrm{nCoV})$ infection is suspected: Interim guidance. 2020. p. 21-21.

15. Januzzi JL. American College of Cardiology. Troponin and BNP use in COVID-19. Cardiology Magazine. 2020;18.

16. Tang N, Li D, Wang X, Sun Z. Abnormal coagulation parameters are associated with poor prognosis in patients 
with novel coronavirus pneumonia. J Thromb Haemost. 2020;18(4):844-847.

17. Stefanini GG, Montorfano M, Trabattoni D, Andreini D, Ferrante G, Ancona M, Metra M, et al. ST-elevation myocardial infarction in patients with COVID-19: clinical and angiographic outcomes. Circulation. 2020;141(25):21132116.

18. Goyal P, Choi JJ, Pinheiro LC, Schenck EJ, Chen R, Jabri A, Satlin MJ, et al. Clinical characteristics of COVID-19 in New York City. N Engl J Med. 2020;382(24):23722374.

19. Guo T, Fan Y, Chen M, Wu X, Zhang L, He T, Wang H, et al. Cardiovascular implications of fatal outcomes of patients with coronavirus disease 2019 (COVID-19). JAMA Cardiol. 2020;5(7):811-818.

20. Mehra MR, Ruschitzka F. COVID-19 illness and heart failure: a missing link? JACC Heart Fail. 2020;8(6):512514.

21. Argulian E, Sud K, Vogel B, Bohra C, Garg VP, Talebi S, Lerakis $\mathrm{S}$, et al. Right ventricular dilation in hospitalized patients with COVID-19 infection. JACC Cardiovasc Imaging. 2020;13(11):2459-2461.

22. Bikdeli B, Madhavan MV, Jimenez D, Chuich T, Dreyfus I, Driggin E, Nigoghossian C, et al. COVID-19 and thrombotic or thromboembolic disease: implications for prevention, antithrombotic therapy, and followup: JACC State-of-the-Art review. J Am Coll Cardiol. 2020;75(23):2950-2973.

23. Wang T, Chen R, Liu C, Liang W, Guan W, Tang R, Tang $\mathrm{C}$, et al. Attention should be paid to venous thromboembolism prophylaxis in the management of COVID-19. Lancet Haematol. 2020;7(5):e362-e363.

24. Garcia S, Albaghdadi MS, Meraj PM, Schmidt C, Garberich R, Jaffer FA, Dixon S, et al. Reduction in ST-Segment elevation cardiac catheterization laboratory activations in the united states during COVID-19 pandemic. J Am Coll Cardiol. 2020;75(22):2871-2872.

25. Alsidawi S, Campbell A, Tamene A, Garcia S. Ventricular septal rupture complicating delayed acute myocardial infarction presentation during the COVID-19 pandemic. JACC Case Rep. 2020;2(10):1595-1598.

26. Mahmud E, Dauerman HL, Welt FGP, Messenger JC, Rao SV, Grines C, Mattu A, et al. Management of acute myocardial infarction during the COVID-19 pandemic: a position statement from the Society for Cardiovascular Angiography and Interventions (SCAI), the American College of Cardiology (ACC), and the American College of Emergency Physicians (ACEP). J Am Coll Cardiol. 2020;76(11):1375-1384.

27. Mehta SR, Granger CB, Boden WE, Steg PG, Bassand JP, Faxon DP, Afzal R, et al. Early versus delayed invasive intervention in acute coronary syndromes. N Engl J Med. 2009;360(21):2165-2175.

28. Han Y, Zeng H, Jiang H, Yang Y, Yuan Z, Cheng X, Jing $Z$, et al. CSC expert consensus on principles of clinical management of patients with severe emergent cardiovascular diseases during the COVID-19 epidemic. Circulation. 2020;141(20):e810-e816.
29. Beigel JH, Tomashek KM, Dodd LE. Remdesivir for the treatment of COVID-19 - preliminary report. Reply. N Engl J Med. 2020;383(10):994.

30. Recovery Collaborative Group, Horby P, Lim WS, Emberson JR, Mafham M, Bell JL, Linsell L, et al. Dexamethasone in hospitalized patients with COVID-19. N Engl J Med. 2021;384(8):693-704.

31. Sanders JM, Monogue ML, Jodlowski TZ, Cutrell JB. Pharmacologic treatments for coronavirus disease 2019 (COVID-19): A Review. JAMA. 2020;323(18):18241836.

32. Hu H, Ma F, Wei X, Fang Y. Coronavirus fulminant myocarditis treated with glucocorticoid and human immunoglobulin. Eur Heart J. 2021;42(2):206.

33. Zhang P, Zhu L, Cai J, Lei F, Qin JJ, Xie J, Liu YM, et al. Association of Inpatient Use of angiotensin-converting enzyme inhibitors and angiotensin II receptor blockers with mortality among patients with hypertension hospitalized with COVID-19. Circ Res. 2020;126(12):16711681.

34. Tisdale JE, Jaynes HA, Kingery JR, Mourad NA, Trujillo TN, Overholser BR, Kovacs RJ. Development and validation of a risk score to predict QT interval prolongation in hospitalized patients. Circ Cardiovasc Qual Outcomes. 2013;6(4):479-487.

35. Chorin E, Dai M, Shulman E. The QT interval in patients with SARS-CoV-2 infection treated with hydroxychloroquine/azithromycin. medRxiv. Preprint posted online, 2020.

36. Saleh M, Gabriels J, Chang D, Soo Kim B, Mansoor A, Mahmood E, Makker P, et al. Effect of Chloroquine, Hydroxychloroquine, and Azithromycin on the Corrected QT Interval in Patients With SARS-CoV-2 Infection. Circ Arrhythm Electrophysiol. 2020;13(6):e008662.

37. McCullough PA, Kelly RJ, Ruocco G, Lerma E, Tumlin J, Wheelan KR, Katz N, et al. Pathophysiological basis and rationale for early outpatient treatment of SARS-CoV-2 (COVID-19) Infection. Am J Med. 2021;134(1):16-22.

38. Abou-Arab O, Huette P, Debouvries F, Dupont H, Jounieaux V, Mahjoub Y. Inhaled nitric oxide for critically ill Covid-19 patients: a prospective study. Crit Care. 2020;24(1):645.

39. Simonovich VA, Burgos Pratx LD, Scibona P, Beruto MV, Vallone MG, Vazquez C, Savoy N, et al. A randomized trial of convalescent plasma in COVID-19 severe pneumonia. N Engl J Med. 2021;384(7):619-629.

40. Libster R, Perez Marc G, Wappner D, Coviello S, Bianchi A, Braem V, Esteban I, et al. Early high-Titer plasma therapy to prevent severe COVID-19 in older adults. N Engl J Med. 2021;384(7):610-618.

41. Kusters PJH, Lutgens E, Seijkens TTP. Exploring immune checkpoints as potential therapeutic targets in atherosclerosis. Cardiovasc Res. 2018;114(3):368-377.

42. Puntmann VO, Carerj ML, Wieters I, Fahim M, Arendt C, Hoffmann J, Shchendrygina A, et al. Outcomes of cardiovascular magnetic resonance imaging in patients recently recovered from coronavirus disease 2019 (COVID-19). JAMA Cardiol. 2020;5(11):1265-1273. 